\title{
Article \\ WisecondorFF: Improved Fetal Aneuploidy Detection from Shallow WGS through Fragment Length Analysis
}

\author{
Tom Mokveld $^{1}$, Zaid Al-Ars ${ }^{2}$, Erik A. Sistermans ${ }^{3}\left(\mathbb{D}\right.$ and Marcel Reinders ${ }^{1, *(D)}$ \\ 1 Delft Bioinformatics Lab, Delft University of Technology, Van Mourik Broekmanweg 6, \\ 2628 XE Delft, The Netherlands; T.O.Mokveld@tudelft.nl \\ 2 Computer Engineering, Delft University of Technology, Mekelweg 4, 2628 CD Delft, The Netherlands; \\ Z.Al-Ars@tudelft.nl \\ 3 Department of Human Genetics and Amsterdam Reproduction \& Development Research Institute, \\ Amsterdam UMC, Vrije Universiteit Amsterdam, Van der Boechorststraat 7, \\ 1081 BT Amsterdam, The Netherlands; e.sistermans@amsterdamumc.nl \\ * Correspondence: M.J.T.Reinders@tudelft.nl
}

Citation: Mokveld, T.; Al-Ars, Z. Sistermans, E.A.; Reinders, M. WisecondorFF: Improved Fetal Aneuploidy Detection from Shallow WGS through Fragment Length Analysis. Diagnostics 2022, 12, 59 https://doi.org/10.3390/ diagnostics 12010059

Academic Editor: Ray Iles

Received: 29 November 2021

Accepted: 21 December 2021

Published: 28 December 2021

Publisher's Note: MDPI stays neutral with regard to jurisdictional claims in published maps and institutional affiliations.

Copyright: (C) 2021 by the authors. Licensee MDPI, Basel, Switzerland. This article is an open access article distributed under the terms and conditions of the Creative Commons Attribution (CC BY) license (https:// creativecommons.org/licenses/by/ $4.0 /)$.

\begin{abstract}
In prenatal diagnostics, NIPT screening utilizing read coverage-based profiles obtained from shallow WGS data is routinely used to detect fetal CNVs. From this same data, fragment size distributions of fetal and maternal DNA fragments can be derived, which are known to be different, and often used to infer fetal fractions. We argue that the fragment size has the potential to aid in the detection of CNVs. By integrating, in parallel, fragment size and read coverage in a within-sample normalization approach, it is possible to construct a reference set encompassing both data types. This reference then allows the detection of CNVs within queried samples, utilizing both data sources. We present a new methodology, WisecondorFF, which improves sensitivity, while maintaining specificity, relative to existing approaches. WisecondorFF increases robustness of detected CNVs, and can reliably detect even at lower fetal fractions $(<2 \%)$.
\end{abstract}

Keywords: non-invasive prenatal testing; cell-free DNA; fragment size; within-sample normalization

\section{Introduction}

Prenatal screening is routinely used to verify and measure the health of the fetus, including the detection of chromosomal CNVs (copy number variations), in a timely manner [1]. Most prenatal screening methods are now non-invasive. Invasive methods typically lead to more conclusive results, but confer a low risk of harming the mother and/or fetus [2-4]. For example, verifying chromosomal aneuploidies through invasive genetic analysis carries a small, but significant, risk of causing fetal miscarriage [5]. The noninvasive methods assess fetal health through indirect means such as through morphological properties using ultrasound scans $[6,7]$, or biochemical markers by sampling the maternal serum $[8,9]$. Generally, such screening methods must be performed at specific stages of the pregnancy [10], and are typically deployed in parallel [5,6], broadening the scope of detectable pathologies [10-12].

Since the discovery of cell-free fetal DNA (cffDNA) within the maternal peripheral bloodstream [13], it has become feasible to measure fetal DNA without invasively intervening [14]. This has opened new possibilities to safely assess fetal health utilizing genetic markers [15], and can be used to detect a wide variety of pathologies caused by events such as chromosomal aneuploidy [14,15], sub-chromosomal CNVs [16], and single-gene mutations [17]. One of the deciding factors to detect ever smaller events is largely bound by the sequencing yield, i.e., the DNA coverage, requiring more sequencing to reliably detect smaller events. The other important factor is the proportion of cffDNA that is mixed within the maternal blood, i.e., the fetal fraction, which normally contributes to $2-20 \%$ of the overall pool of available DNA $[18,19]$, and increases slightly as the pregnancy progresses. 
The combination of available coverage, the fetal fraction, and the size of the CNV determine the reliability of detecting any given aneuploidy.

In clinical practice, NGS-based non-invasive prenatal screening (NIPT) with cffDNA typically utilizes shallow whole-genome sequencing (WGS) to remain economical and accessible for mass screening [20]. Such low sequencing yield leads to practical limits when detecting events, and often means that only high sensitivity and specificity is possible for larger events such as chromosomal aneuploidies. Therefore, NIPT typically tests for common trisomies, such as 21, 18, and 13 [21], since accuracy degrades too much for sub-chromosomal CNVs smaller than 5-10 megabases (Mb) [22-24]. Nevertheless, even for larger events, care should be taken for discordant results caused by biological phenomena such as placental mosaicism or maternal copy number variation $[25,26]$. While the NIPT field is dominated by methods utilizing WGS, there are also those that use RNAseq [27], methylation profiles [28], SNPs [29], or haplotyping [30]. Each of which can fulfill an effective role in detecting specific events, especially when such data sources are integrated [27].

Most low sequencing yield NIPT methods utilize similar steps to detect events [31-33], starting with DNA sequencing, followed by mapping sequencing reads to a reference genome, and finally detecting whether the observed read coverage exceeds expectations based on a reference baseline. As the coverage is extremely low, the detection does not happen on a per nucleotide level, but, instead, the genome is discretized into larger equally sized regions or bins (often $250 \mathrm{~kb}$ to $1 \mathrm{Mb}$ ) in which read counts are aggregated to obtain a signal which can be compared to a baseline. Such a baseline signal, also known as a reference set, can be derived from a collection of healthy samples [34]. Although this can be effective, there are some drawbacks. For example, the experimental conditions of the sample and the healthy samples used for establishing the baseline should be identical in order to eliminate any technical biases that can confound the detection, otherwise one easily gets false positives or negatives [35]. Resequencing the baseline together with a new sample would protect towards these biases but is extremely costly.

An alternative approach to define the baseline would be to compare the observed read count with expectations derived from the sample itself. Within such a setting, a region should be compared to regions on other chromosomes that were found to behave similarly across a healthy panel. Having generated a map of similarly behaving regions from the set of healthy samples, any NIPT sample can be tested exploiting this map without (re)using the set of healthy samples. This setting is effective when the number of events is typically limited to a subset of a chromosome at a time, and is successfully adopted in clinical practice using methods such as Wisecondor and WisecondorX [35,36].

It is known that the fetal DNA fragment size is shorter than that of the maternal fragments [30]. This observation has been exploited in earlier work to predict the fetal fraction within cfDNA samples, i.e., the relative proportion of maternal and fetal DNA present within a sample $[37,38]$. The fragment size of cffDNA can be inferred from pairedend reads, but also by other approaches for instance, using methylation profiles [39] or based on read abundance approaches within and outside of the nucleosomes [40]. As the fetal fragment size is shorter than the maternal fragment size, this can potentially also be used to infer the abundance of fetal reads in any given genomic region. Namely, if a fetus is affected by a trisomy, an overall lower fragment size on the affected chromosome is expected as compared to the unaffected chromosomes. Our aim was to enrich the current NIPT testing procedure with this available data, as it is currently the norm to generate paired-end reads.

We introduce WisecondorFF, a methodology that detects chromosomal in the fetus from cfDNA that combines estimates based on read coverage as well as fragment size statistics. To control for variations, fragment size statistics are derived using a similar within-sample normalization approach as is exploited by current read-count procedures such as Wisecondor. We show that chromosomal CNVs can indeed be detected from inferred fragment sizes across the genome and that, when combined with read coverage, 
leads to improved accuracy and robustness of the NIPT procedure. As such WisecondorFF is interesting for clinical practice as the data is readily available in most clinical diagnostics facilities, as it only relies on paired-end sequencing of the DNA in maternal serum.

\section{Materials and Methods}

\subsection{Sample Specification, Read Alignment, and Filtering}

Samples were generated as part of the Dutch TRIDENT study [41]. DNA isolation, library preparation and paired end sequencing (36 bp) were performed using the Illumina VeriSeq1 sequencing protocol, according to the recommendations of the supplier (Illumina, San Diego, CA, USA). Analysis was performed by both the Veriseq algorithm (which only detects trisomies 21, 13 and 18), and by Wisecondor, which also detects other trisomies and smaller events. For this study we selected 526 samples, of which 401 had no detected chromosomal CNVs and were used as negative controls. The remaining 125 samples all tested positive for T21. The mean depth of coverage is 0.258 and 0.256 , respectively, for the negative and positive samples. All read data were similarly processed and aligned to the hg19 human reference genome (excluding decoy sequences) using BWA-0.7.17 mem [42]. Paired reads are filtered according to the following criteria: (1) reads must be in the correct position/orientation for pairing; (2) only primary alignments are considered; (3) alignments must exceed a minimum mapping quality of at least 1 ; (4) every read should have a unique starting location (Supplementary Figure S1). Alignments were compressed and left unfiltered, as all methods perform internal quality control on alignments.

\subsection{Fragment Sizes}

When quantifying the read coverage, reads are assigned to their respective region according to their starting position, whereas for read-pairs, we adopt their midpoint, i.e., the average of the starting positions. Fragment sizes were determined by the difference between the starting points of the paired reads. They were distributed with a mean of $173 \mathrm{bp}$ and standard deviation of $56 \mathrm{bp}$. Fragment sizes above $300 \mathrm{bp}$ were ignored as these were found to be uninformative when distinguishing between negative and positive samples. Per sample regions of interest were filtered to enable a more reliable estimate on the fragment size distribution by a lower bound on the minimal number of reads and an upper bound which is dependent on the normalized read coverage (Supplementary Figure S2).

\subsection{Preprocessing and Reference Set Construction}

Read coverage is normalized across all samples. The genome is divided into regions of $5 \mathrm{~kb}$ and these regions were consequently scaled up to $250 \mathrm{~kb}, 500 \mathrm{~kb}, 750 \mathrm{~kb}, 1 \mathrm{Mb}, 5 \mathrm{Mb}$, and $10 \mathrm{Mb}$ to test resolution dependent differences. Region sizes smaller than $250 \mathrm{~kb}$ were excluded given that the read count and/or fragment size signals become too noisy at the specified sequencing yield. We did not predefine a list of genomic regions to exclude from the analysis. Instead, the within-sample methods define those based on the normalized read counts during reference set construction, with negligible differences between methods. Such uninformative regions are masked and typically appear at centromeres or highly repetitive locations where insufficient reads can be aligned. Next technical biases (e.g., GC bias) are removed by training PCAs on the negative controls (Supplementary Figure S3). Note that this happens in parallel, hence two PCA mappings exist (one for each datatype), which are saved and applied to any sample that the reference is queried with.

\subsection{WisecondorFF}

WisecondorFF builds upon the same within-sample testing methodology as Wisecondor and WisecondorX $[35,36]$ : constructing a reference set of similarly behaving regions as derived from control samples, and then processing each region in a new sample given this reference set. Hence, creating the reference set lies at the core of the methodology, which is based on the observation that the (ab)normality of a region on one chromosome can be 
judged relative to the behavior of similarly behaving regions (the references) in the control samples on other chromosomes. The similarity metric can be data-type dependent. For read count data, we follow Wisecondor which uses the Euclidean distance between the two vectors of read count in the two regions of consideration across the control samples. For each region, all regions on the other chromosomes are ranked based on the similarity metric, and the top $K$ (here $K=300$ ) regions are selected to be the reference set of regions for the region of consideration. Regions are additionally weighted according to reliability, based on the calculated distances. Doing this for all regions creates the complete reference set.

For fragment size data, the similarity metric between two regions is the Euclidean distance between the two vectors of mean fragment sizes within a region for each control sample. We experimented with different summarizations of the fragment distributions in a region, such as the median (being less predictive, Supplementary Figure S4), or measures that directly capture the difference between two distributions, such as the Jensen-Shannon divergence distance (symmetrized Kullback-Leibler divergence). The latter was, however, not feasible due to the amount of noise present within the distributions (full details in Supplemental Section S7).

When testing a sample for CNVs, first, a Z-score for each (query) region and each data type is calculated separately. This Z-score can be calculated from the observed measurement (here either the read count or fragment size) in the query region with respect to the mean and standard deviation of that measurement calculated across the reference regions for the query region within that same sample. The data type specific score (of each region) can be joined into a single score by Fisher's averaging. Next, the scores per region are used to find stretches of affected regions through segmentation. Here, we follow the methodology of WisecondorX [36] and use CBS (Circular Binary Segmentation) [43] to segment and finally obtain Z-scores of detected events.

\subsection{Fetal Fraction Estimation}

Sample fetal fraction was estimated using SeqFF [38], this method uses a pre-trained multivariate model that was trained on the per region stratified autosomal read counts from WGS paired-end sequencing of cfDNA from maternal plasma. The average fetal fraction is $7.5 \%$ and ranges between $1.48 \%$ and 19.15\% (Supplementary Figure S5).

\section{Results}

\subsection{Fragment Size Distributions Do Differ between T21 Affected and Unaffected Samples}

To determine whether the fragment size can indeed be indicative of samples with a trisomy, we, first, studied the fragment size distribution within our cohort of 526 samples, across chromosome 21, for 125 samples with a trisomy of chromosome 21 as well as 401 samples having no trisomy (negative samples). Figure 1a shows that indeed, on average, a distribution shift of $\sim 1.52$ bp to shorter fragments can be observed for the T21 samples. Note that the fragment size distributions for individual samples vary considerably, likely caused by differences in fetal fraction and/or technical noise. While Figure 1a shows a shift in the chromosome-wide fragment size distribution, we can observe a similar shift in distributions when we consider smaller regions across chromosome 21, as shown in Figure 1b. These differences become, however, less noticeable when the region size decreases as fewer reads fall within a region, resulting in more noisy estimates of these distributions. With an average sequence coverage of $0.25 \times$, we found that a minimum region size of $250 \mathrm{~kb}$ was required to estimate the fragment size distributions with sufficient robustness (Supplementary Figure S6). 


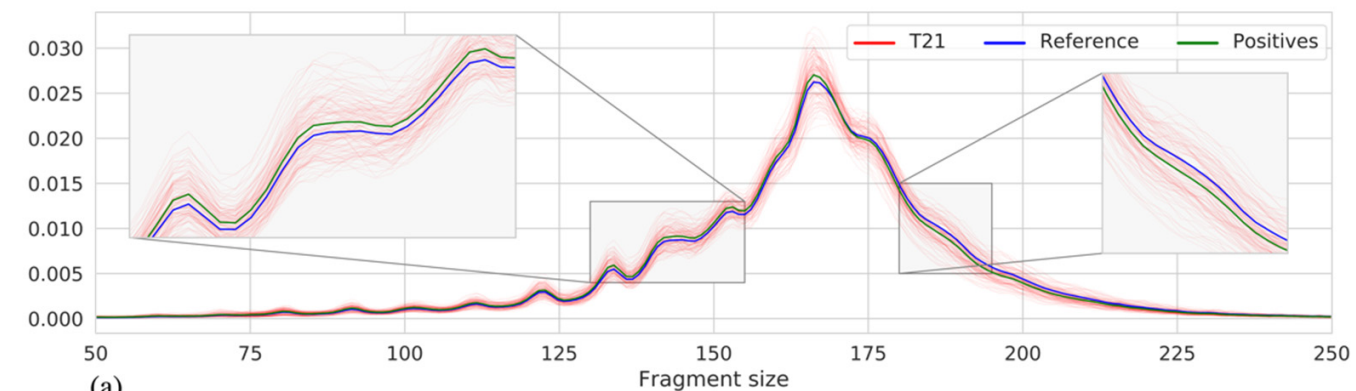

(a)

Fragment size

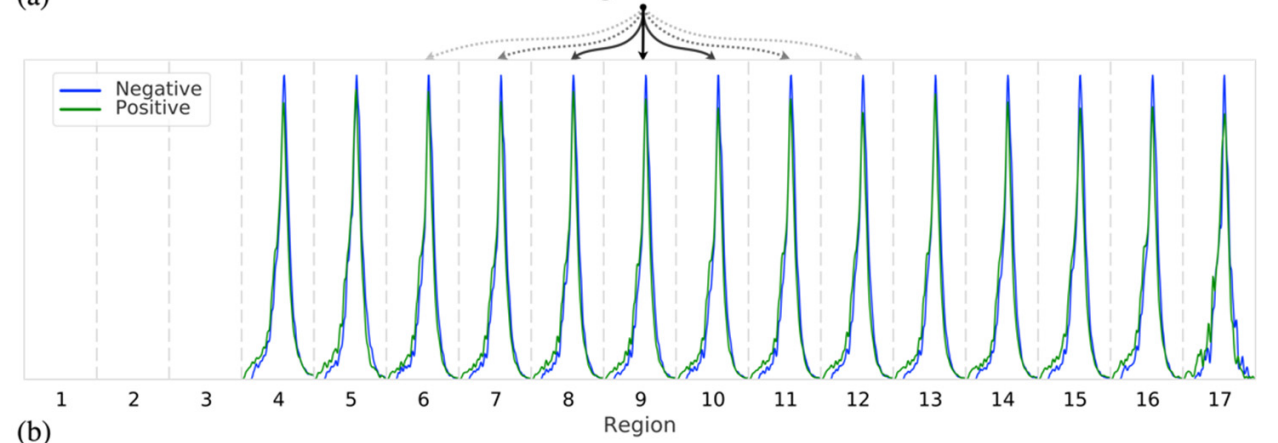

Figure 1. (a) The fragment size distributions of chromosome 21 across samples: individual T21 positive samples (red), the mean of all 125 T21 positive samples (green), and the mean of all 401 negative samples (blue). (b) Discretized representation of chromosome 21 into $3 \mathrm{Mb}$ sized regions, per region fragment size distributions are shown (as in (a)) of two samples, with similar fetal fraction, one negative (blue) and one T21 positive (green).

\subsection{WisecondorFF Detects Aneuploidy Most Robustly}

Within our cohort of 526 samples, we investigated the detection of common whole chromosome aneuploidies using six different approaches. The first three make use of WisecondorFF (Section 2.4), denoted as WcrFF, and detect the presence of a CNV event from read count frequencies $\left(\mathrm{WcrFF}{ }^{\mathrm{RC}}\right)$, fragment size statistics $\left(\mathrm{WcrFF}^{\mathrm{FS}}\right)$, or both $\left(\mathrm{W} c r F F^{\mathrm{RC} \& \mathrm{FS}}\right)$. Two other approaches are the latest versions of Wisecondor (Wcr) [35] and WisecondorX (WcrX) [36]. Finally, we included one method that does not utilize a within sample testing approach to detect CNVs: CNVkit [44], a general purpose CNV detector, which we here use as a baseline.

Nearly all 125 T21s are detected by all methods, where we consider only events when the segments are larger than $10 \mathrm{Mb}$ with Z-scores $\geq 5$, Table 1:I. The performance of all methods is relatively stable across the range of selected region sizes, with exception of $\mathrm{WcrFF}^{\mathrm{FS}}$, which continuously improves as region size increases, and to a lesser extent in $\mathrm{WcrFF}^{\mathrm{RC}}$ and $\mathrm{WcrX}$ at $10 \mathrm{Mb}$, with performance sharply dropping. The former can be attributed to the increased noise in the fragment size signal, which we also encountered when attempting to call events based on the fragment size distributions of each region rather than their means (Supplementary Figure S7). The latter is likely caused by the CBS algorithm used by both methods. Note that only WcrFF ${ }^{R C \& F S}$ (at $\left.750 \mathrm{~kb}\right)$ can detect all expected trisomies and has near optimal and stable performance within the other region sizes. The baseline, CNVkit, is competitive with the other methods, which is expected given the relative ease of detecting T21 events. 
Table 1. The number of detected events in the $125 \mathrm{~T} 21$ positive samples for the six different tested methods (rows). An event is detected when $\mathbf{I}$ : a segment is larger than $10 \mathrm{Mb}$ with a Z-score $\geq 5$ or II: a segment larger than $1 \mathrm{Mb}$ with $\mid \mathrm{Z}$-score $\mid \geq 5$. The number of samples for which an event is detected on chromosome 21 is given for different reference region sizes (columns). Between parenthesis, we note the number events detected on one of the other chromosomes.

\begin{tabular}{|c|c|c|c|c|c|c|c|}
\hline & & $250 \mathrm{~kb}$ & $500 \mathrm{~kb}$ & $750 \mathrm{~kb}$ & $1 \mathrm{Mb}$ & $5 \mathrm{Mb}$ & $10 \mathrm{Mb}$ \\
\hline \multirow[b]{2}{*}{$\mathrm{WcrFF}^{\mathrm{FS}}$} & $\mathrm{I}$ & $71(0)$ & $77(0)$ & $83(0)$ & $86(6)$ & $92(14)$ & $106(5)$ \\
\hline & II & $71(0)$ & $77(0)$ & $83(0)$ & $87(11)$ & $92(14)$ & $106(5)$ \\
\hline \multirow{2}{*}{ WcrFF ${ }^{R C}$} & $\mathrm{I}$ & $122(0)$ & $122(1)$ & $122(1)$ & $120(2)$ & $121(0)$ & $103(0)$ \\
\hline & II & $122(3)$ & $122(2)$ & $122(1)$ & $120(2)$ & $121(0)$ & $103(0)$ \\
\hline \multirow{2}{*}{ WcrFF ${ }^{R C \& F S}$} & I & $124(17)$ & $124(14)$ & $125(13)$ & $124(14)$ & $124(6)$ & $122(3)$ \\
\hline & II & $125(18)$ & $125(15)$ & $125(17)$ & $125(14)$ & $124(6)$ & $124(3)$ \\
\hline \multirow{2}{*}{ Wcr } & $\mathrm{I}$ & $123(162)$ & $124(78)$ & $123(34)$ & $123(27)$ & $122(9)$ & $121(8)$ \\
\hline & II & $124(471)$ & $124(285)$ & $123(119)$ & $123(215)$ & $122(16)$ & $121(8)$ \\
\hline \multirow[b]{2}{*}{ WcrX } & I & $122(1)$ & $122(0)$ & $120(1)$ & $121(1)$ & $120(1)$ & $103(0)$ \\
\hline & II & $122(4)$ & $122(0)$ & $120(1)$ & $121(1)$ & $120(1)$ & $103(0)$ \\
\hline \multirow{2}{*}{ CNVkit } & I & \multicolumn{6}{|c|}{$120(375)$} \\
\hline & II & \multicolumn{6}{|c|}{$124(896)$} \\
\hline
\end{tabular}

Although the majority of all expected trisomies were detected by each of the methods, this should be put into context with any additional findings, i.e., false positives. In Table 1, we also summarize the detection of events on chromosomes other than 21 in parenthesis. Generally, sensitivity increases when a smaller region size is chosen, which is especially true for Wcr. WcrFF ${ }^{\text {RC\&FS }}$ makes far fewer false positive calls than Wcr at any resolution, while detecting slightly more than $\mathrm{WcrX}$. When we change the constraints of accepting an event to segments larger than $1 \mathrm{Mb}$ with $\mid \mathrm{Z}$-score $\mid \geq 5$, allowing smaller events to be called (Table 1:II). Wcr calls significantly more false positives (+247.17\%) while still not detecting all expected T21 events, whereas $W_{c r F F}{ }^{R C \& F S}$ is now able to detect all expected events at nearly every resolution, with only a modest increase in false positives (+8.95\%).

\subsection{WisecondorFF Has Most Power}

Next, we investigated the Z-scores generated by the different within-sample methods, since higher Z-scores indicate more power to detect an event. Per region on chromosome 21, we calculated the average Z-scores across all T21 positive samples. From Figure 2a, we can observe that the highest Z-scores are found by the $\mathrm{WcrFF}^{\mathrm{RC} \& \mathrm{FS}}$ method when considering region sizes of $750 \mathrm{~kb}$. Note that the $Z$-scores for $W_{c r F F}{ }^{R C}$ drop, behaving very similarly to WcrX, and drop even more dramatically with $\mathrm{WcrFF}^{\mathrm{FS}}$, which also performs worse than Wcr. Similar results are obtained across other region scales. The distribution of average Z-scores per region, as shown in Figure 2b, also display the shift towards larger Z-scores for WcrFFRC\&FS.

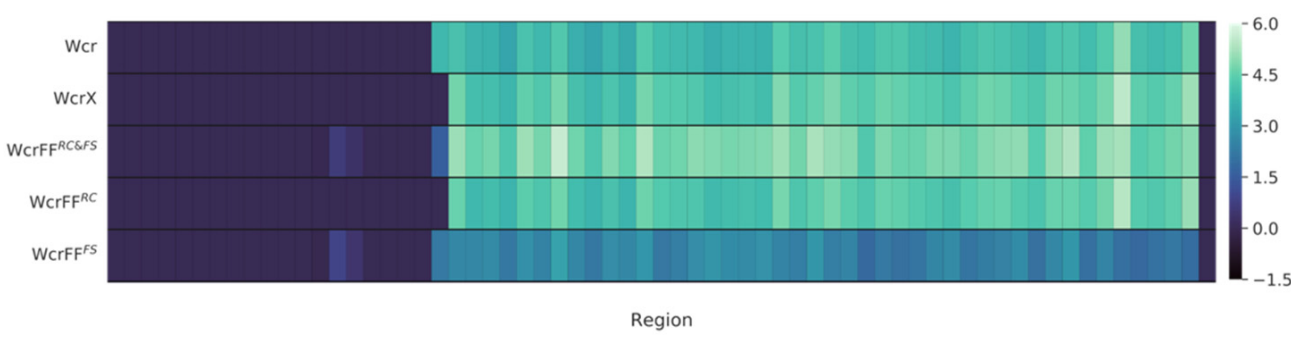

(a)

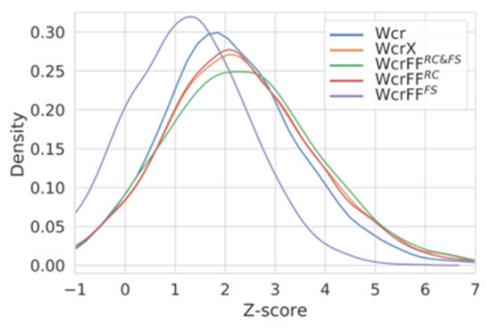

(b)

Figure 2. (a) Heatmap of the Z-scores averaged across all T21 positive samples, shown per $750 \mathrm{~kb}$ sized region (columns) on chromosome 21 for the different methods (rows). (b) Z-score distributions of all T21 positive samples on chromosome 21, with zero value Z-scores filtered out. 


\subsection{The Power to Detect Trisomies Comes from Combining Read Count and Fragment Size Data}

Next, we quantified the differences between Z-scores derived from either fragment size or read coverage as well as the combined approach. Figure 3 shows the average Z-scores across chromosome 21 for each of the 526 samples for $1 \mathrm{Mb}$ sized regions for the methods. As expected, the negative samples have mean Z-scores that are closely centered around zero. Overall, we can see that the Z-score magnitude of events detected by $\mathrm{WcrFF}^{\mathrm{RC}}$ are larger than those detected by $\mathrm{W}_{\mathrm{crFF}} \mathrm{FS}^{\mathrm{F}}$. Hence, the fragment size by itself is not as reliable or powerful as the read count. However, when combining the two measures, as in $\mathrm{WcrFF}^{\mathrm{RC} \& \mathrm{FS}}$, it becomes possible to separate all negative and T21 positive samples, without detecting false positives. Additionally, the Z-score magnitude for the T21 samples is generally greater when combining both inputs compared to using only the read coverage.

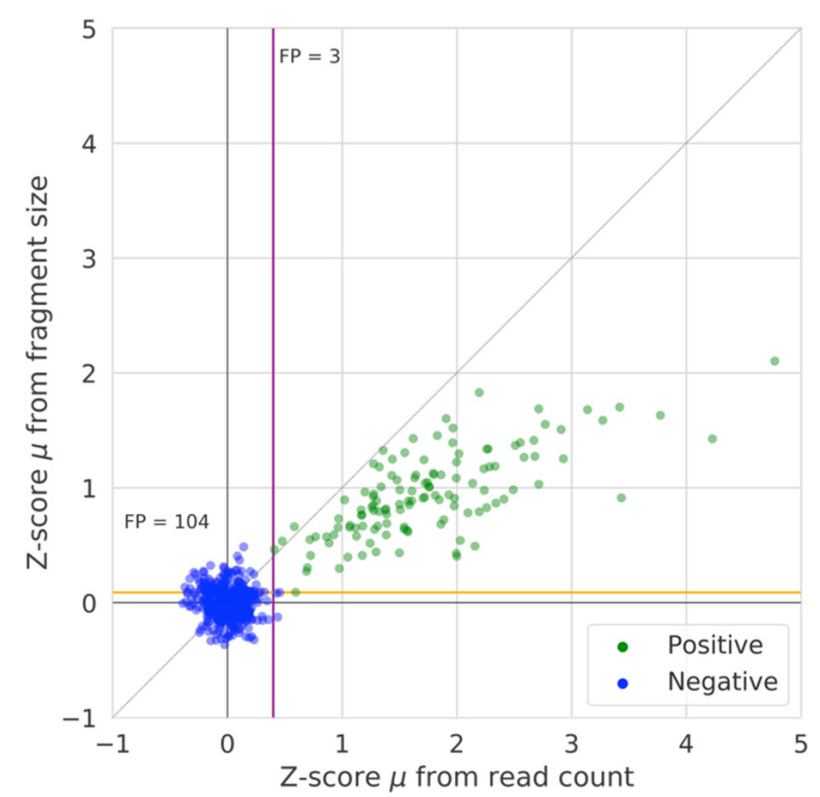

(a)

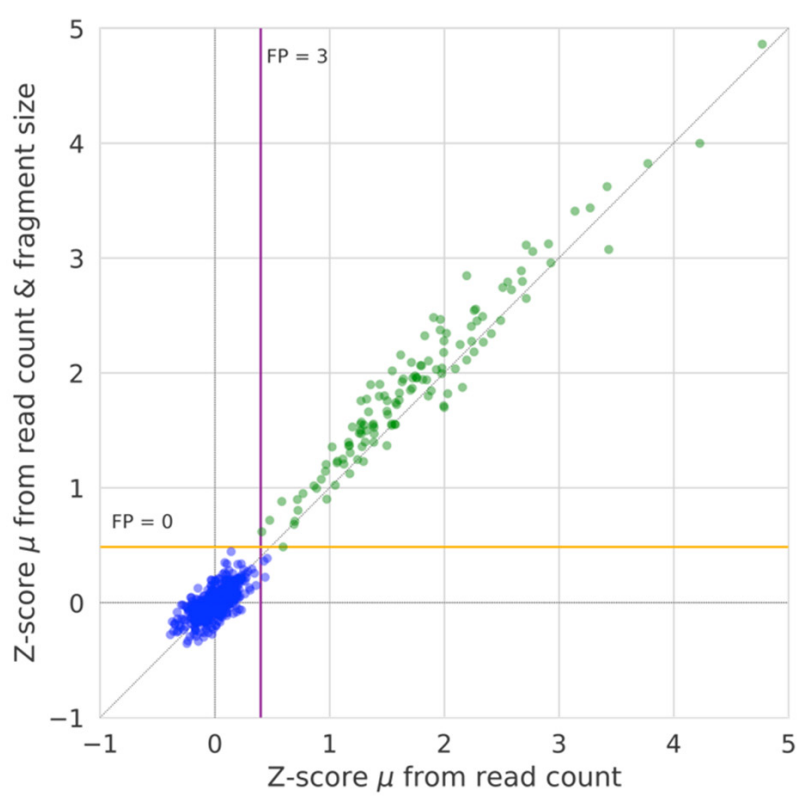

(b)

Figure 3. The average $\mathrm{Z}$-score of all $1 \mathrm{Mb}$ sized regions on chromosome 21 for all 526 samples for: (a) $\mathrm{WcrFF}^{\mathrm{RC}}$ compared to $\mathrm{WcrFF}^{\mathrm{FS}}$, and (b) $\mathrm{WcrFF}^{\mathrm{RC}}$ compared to $\mathrm{WcrFF}{ }^{\mathrm{RC} \& F S}$. The colored lines denote the Z-score cutoff boundaries that would capture all T21 positive samples for either method (purple for read count and orange for fragment size and fragment size \& read count), annotations denote the number of false positives (FP) given these cut-offs.

\subsection{Increased Power of WisecondorFF Allows to Detect CNVs at Lower Fetal Fractions}

As a NIPT test may be performed at different stages of the pregnancy, we were interested in how the methods perform at different fractions of cffDNA available in the maternal blood plasma. Hereto, we estimated the fetal fractions of the 125 samples (Section 2.5.) and compared those to the Z-scores of detected events on chromosome 21 (Figure 4). We show that $\mathrm{W} c r F F^{R C \& F S}$ assigns greater Z-scores in nearly all cases and at all fetal fraction ranges. Interesting is the detection of a duplication event in a sample with a sub $2 \%$ fetal fraction, which was undetectable by either Wcr or WcrX. 


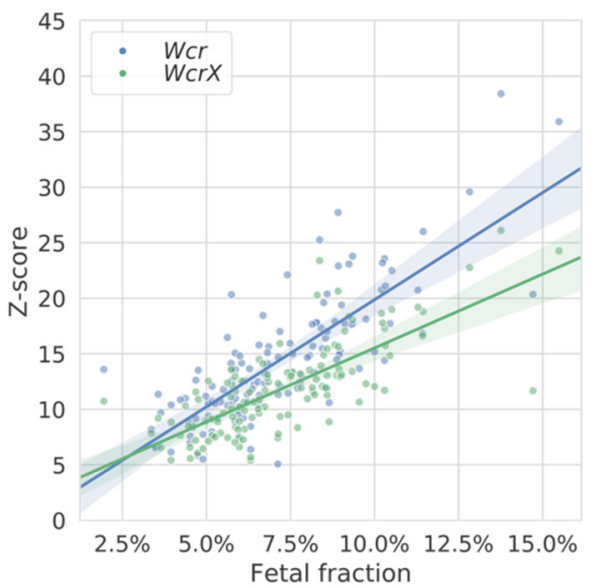

(a)

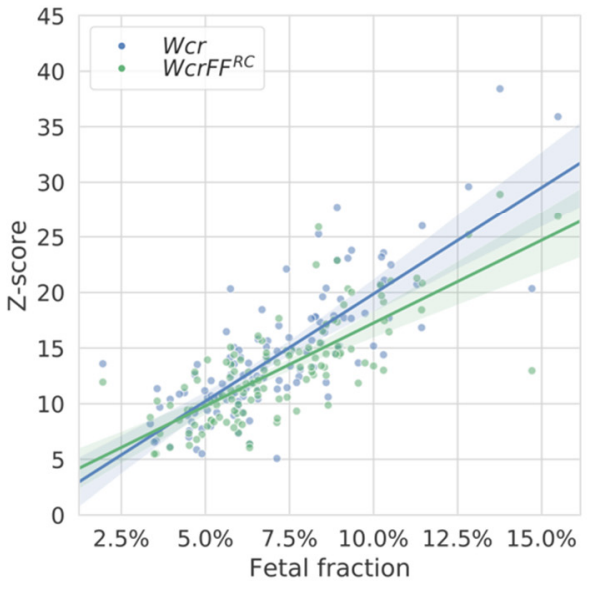

(c)

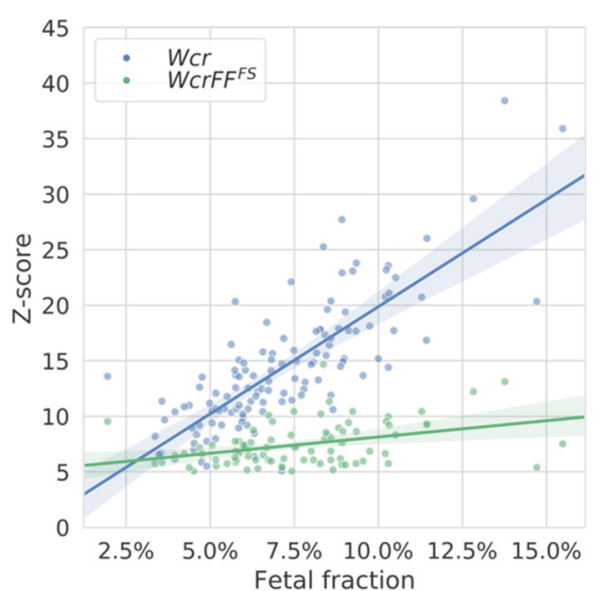

(b)

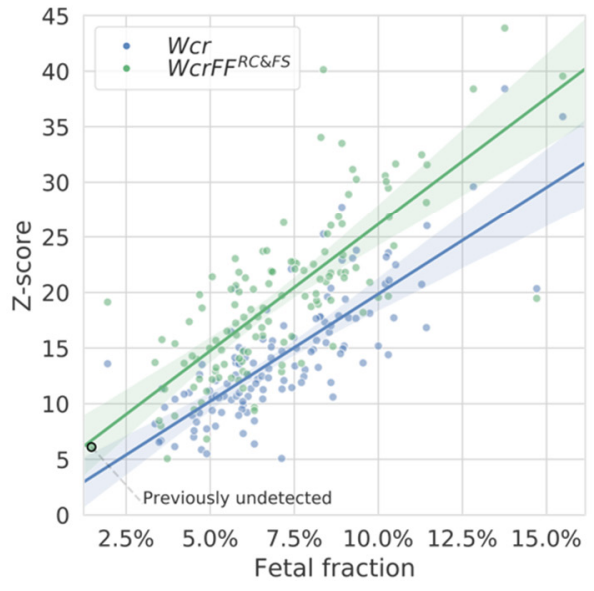

(d)

Figure 4. Z-scores of duplication events on chromosome 21 (an event is detected when a segment is larger than $10 \mathrm{Mb}$ with a Z-score $\geq 5$ ) as detected by the different methods in the $125 \mathrm{~T} 21$ samples with respect to the estimated fetal fractions of each sample. In each panel one of the methods is compared with WCR (in blue): (a) WcrX, (b) $\mathrm{WcrFF}^{\mathrm{FS}}$, (c) $\mathrm{WcrFF}^{\mathrm{RC}}$, and (d) $\mathrm{WcrFF}^{\mathrm{RC} \& F S}$ (all in green). Region size was $750 \mathrm{~kb}$. Each point corresponds to an event within a sample.

\section{Discussion}

Detecting CNVs in heterogeneously mixed WGS data remains challenging and is a point of ongoing research within the NIPT field, but also in adjacent fields such as cancer diagnostics from cell free tumor DNA [45]. With NIPT, one must deal with a mixture of maternal and fetal DNA in which the fetal, and thus the potentially affected part, occurs at a far smaller concentration. Moreover, when sampling at different stages of the pregnancy, different levels of fetal fraction are encountered. In addition, differences in sample preparation and sequencing can introduce sufficient noise to mask true variations within the sample. When utilizing reference-based methods that directly compare a sample signature to a reference signal, such noise is difficult to cope with. A within-sample normalization method circumvents these issues of experimental noise.

Generally speaking, NIPT methods utilizing WGS data exploit the relative frequencies of reads aligned to a reference genome. This is not without reason as the read coverage is a powerful predictor for the presence of a CNV. However, other data types are known to be informative for $\mathrm{CNV}$ detection. One source of information that can be readily derived from paired-end reads is the cfDNA fragment size. Although, it is general knowledge that this size differs between maternal and fetal cfDNA, in NIPT CNV detection applications 
this information is not utilized, even though most sequencing for NIPT nowadays is done using short paired-end sequencing.

We have shown that, both on a whole-chromosomal and a sub-chromosomal level, the fragment size distributions are indeed shifted in a detectable way when dealing with chromosomal CNVs in the fetal DNA. To detect these events, we introduced WisecondorFF a within-sample normalization method that utilizes both the relative frequencies of aligned reads as well as the fragment size to reliably detect chromosomal CNVs.

We noted that our presented method, WisecondorFF (WcrFF ${ }^{R C \& F S}$ ), which utilizes both read count and fragment size statistics, make several improvements relative to other methods. Namely WisecondorFF is more sensitive than Wisecondor and WisecondorX, while being more selective, detecting fewer false positives. We found that WisecondorFF is more robust than others by assigning a higher certainty to any detected events at any fetal fraction. This leads to a benefit for WisecondorFF where calls that were previously indistinguishable from the background signal are now more likely to be detected. By relating the Z-scores of detected events with the (estimated) fetal fractions, we found that WisecondorFF can detect events at lower fetal fractions. While WisecondorFF had the best performance with a region size of $750 \mathrm{~kb}$, we showed that the fragment size performs better at larger region scales. Currently, the fragment size and read coverage are integrated at the same scales. However, it would be possible to use asymmetrical region sizes across the different data types for better performance. At this time, WisecondorFF does not build gender specific reference sets, like WisecondorX does, and can, therefore, not detect $\mathrm{CNVs}$ on sex chromosomes. We have currently limited the use of additional information exclusively to the fragment size. However, we do believe that any type of data that can be discretized across genomic regions could potentially be integrated within a parallel within-sample normalization framework as we have shown.

By more fully exploiting the information available in current sequencing technology, we have shown that it is possible to achieve better performance within a low coverage NIPT setting by exploiting fragment size information. This opens other applications that can relate to differences in fragment size such as in cancer diagnostics or for other types of data integration within the NIPT process.

Supplementary Materials: The following are available online at https:/ / www.mdpi.com/article/10 .3390 / diagnostics12010059/s1, Figure S1: 100 bp region stratified read coverage ( 0.25x) of chromosome 1 in a single sample, Figure S2: Per-region coverage distribution plots, Figure S3: Genome-wide GC content with respect to the read count and the fragment/insert size respectively, Figure S4: The average Z-score of all regions ( $1 \mathrm{Mb}$ size) on chromosome 21 for all 526 (positive and negative) samples comparing $\mathrm{WcrFF}^{\mathrm{RC}}$ and $\mathrm{WcrFF}^{\mathrm{RC} \& \mathrm{FS} m e d i a n}$, Figure S5: Fetal fraction values for all 526 samples as estimated by SeqFF, Figure S6: Per region fragment size distribution of chromosome 1 as summarized by the mean and standard deviation across different region scales, Figure S7: The average Z-score of all regions ( $1 \mathrm{Mb}$ region size) on chromosome 21 for all 526 samples for $\mathrm{WcrFF}^{\mathrm{RC}}$ compared to WcrFF ${ }^{\mathrm{FS} \_J S D}$, Note S7: Reference set construction by comparison of fragment size distributions.

Author Contributions: T.M. and M.R. conceived and supervised the study. T.M. developed and implemented the method, and performed the computational experiments. T.M. and M.R. interpreted the results and adapted the methodologies. Z.A.-A. overlooked the computational aspects. E.A.S. overlooked the biological implications. T.M. wrote the manuscript with input from all other authors. All authors have read and agreed to the published version of the manuscript.

Funding: This work is being funded by the Delft Data Science Center of the Delft University of Technology, which has no role in the design and execution of the study as well as the interpretation of the data and writing of the manuscript.

Institutional Review Board Statement: The study was conducted according to the guidelines of the Declaration of Helsinki, and approved by the Institutional Review Board (or Ethics Committee) of VU University Medical Center Amsterdam (protocol code 2021.0515-DISTIL, date of approval 21-09-2021).

Informed Consent Statement: Informed consent was obtained from all subjects involved in the study. 
Data Availability Statement: WisecondorFF is an open source tool. User-manual and software are freely available at: https:/ / github.com/tomokveld/WisecondorFF last accessed on 29 November 2021.

Conflicts of Interest: The authors declare no conflict of interest.

\section{References}

1. Nshimyumukiza, L.; Menon, S.; Hina, H.; Rousseau, F.; Reinharz, D. Cell-free DNA noninvasive prenatal screening for aneuploidy versus conventional screening: A systematic review of economic evaluations. Clin. Genet. 2018, 94, 3-21. [CrossRef] [PubMed]

2. Saura, R.; Spalova, I.; Taine, L.; Horovitz, J.; Grison, O.; Maugey, B.; Smoleniec, J.; James, D.; Verma, R. Evaluation of chorion villus sampling. Lancet 1991, 338, 449-450. [CrossRef]

3. Papp, C.; Papp, Z. Chorionic villus sampling and amniocentesis: What are the risks in current practice? Curr. Opin. Obstet. Gynecol. 2003, 15, 159-165. [CrossRef]

4. Saura, R.; Roux, D.; Taine, L.; Maugey, B.; Laulon, D.; Laplace, J.; Horovitz, J.; Bombard, A.; Carter, S.; Nitowsky, H.; et al. Early amniocentesis versus chorionic villus sampling for fetal karyotyping. Lancet 1994, 344, 825-826. [CrossRef]

5. $\quad$ Dondorp, W.; De Wert, G.; Bombard, Y.; Bianchi, D.W.; Bergmann, C.; Borry, P.; Chitty, L.S.; Fellmann, F.; Forzano, F.; Hall, A.; et al. Non-invasive prenatal testing for aneuploidy and beyond: Challenges of responsible innovation in prenatal screening. Summary and recommendations. Eur. J. Hum. Genet. 2015, 23, 1438-1450. [CrossRef] [PubMed]

6. Colmant, C.; Morin-Surroca, M.; Fuchs, F.; Fernandez, H.; Senat, M.V. Non-invasive prenatal testing for fetal sex determination: Is ultrasound still relevant? Eur. J. Obstet. Gynecol. Reprod. Biol. 2013, 171, 197-204. [CrossRef] [PubMed]

7. Salomon, L.; Alfirevic, Z.; Da Silva Costa, F.; Deter, R.; Figueras, F.; Ghi, T.; Glanc, P.; Khalil, A.; Lee, W.; Napolitano, R.; et al. ISUOG practice guidelines: Ultrasound assessment of fetal biometry and growth. Ultrasound Obstet. Gynecol. 2019, 53, 715-723. [CrossRef]

8. Catic, A.; Gurbeta, L.; Kozaric, A.; Mehmedbasic, S.; Badnjevic, A. Application of Neural Networks for classification of Patau, Edwards, Down, Turner and Klinefelter Syndrome based on first trimester maternal serum screening data, ultrasonographic findings and patient demographics. BMC Med. Genom. 2018, 11, 1-12. [CrossRef]

9. Alleman, B.W.; Smith, A.R.; Byers, H.M.; Bedell, B.; Ryckman, K.K.; Murray, J.C.; Borowski, K.S. A proposed method to predict preterm birth using clinical data, standard maternal serum screening, and cholesterol. Am. J. Obstet. Gynecol. 2013, 208, 472.e1-472.e11. [CrossRef]

10. Kaimal, A.J.; Norton, M.E.; Kuppermann, M. Prenatal Testing in the Genomic Age: Clinical Outcomes, Quality of Life, and Costs. Obstet. Gynecol. 2015, 126, 737-746. [CrossRef]

11. Larion, S.; Warsof, S.L.; Romary, L.; Mlynarczyk, M.; Peleg, D.; Abuhamad, A.Z. Association of Combined First-Trimester Screen and Noninvasive Prenatal Testing on Diagnostic Procedures. Obstet. Gynecol. 2014, 123, 1303-1310. [CrossRef] [PubMed]

12. McLennan, A.; Palma-Dias, R.; da Silva Costa, F.; Meagher, S.; Nisbet, D.L.; Scott, F. Noninvasive Prenatal Testing in Routine Clinical Practice-an Audit of Nipt and Combined First-Trimester Screening in an Unselected Australian Population. Aust. N. Z. J. Obstet. Gynaecol. 2016, 56, 22-28. [CrossRef]

13. Lo, Y.M.D.; Corbetta, N.; Chamberlain, P.F.; Rai, V.; Sargent, I.L.; Redman, C.W.; Wainscoat, J.S. Presence of fetal DNA in maternal plasma and serum. Lancet 1997, 350, 485-487. [CrossRef]

14. Sehnert, A.J.; Rhees, B.; Comstock, D.; De Feo, E.; Heilek, G.; Burke, J.; Rava, R.P. Optimal Detection of Fetal Chromosomal Abnormalities by Massively Parallel DNA Sequencing of Cell-Free Fetal DNA from Maternal Blood. Clin. Chem. 2011, 57, 1042-1049. [CrossRef]

15. Benn, P.; Cuckle, H.; Pergament, E. Non-invasive prenatal testing for aneuploidy: Current status and future prospects. Ultrasound Obstet. Gynecol. 2013, 42, 15-33. [CrossRef] [PubMed]

16. Neofytou, M.C.; Tsangaras, K.; Kypri, E.; Loizides, C.; Ioannides, M.; Achilleos, A.; Mina, P.; Keravnou, A.; Sismani, C.; Koumbaris, G.; et al. Targeted capture enrichment assay for non-invasive prenatal testing of large and small size sub-chromosomal deletions and duplications. PLoS ONE 2017, 12, e0171319. [CrossRef]

17. Skrzypek, H.; Hui, L. Noninvasive prenatal testing for fetal aneuploidy and single gene disorders. Best Pract. Res. Clin. Obstet. Gynaecol. 2017, 42, 26-38. [CrossRef] [PubMed]

18. Canick, J.A.; Palomaki, G.E.; Kloza, E.M.; Messerlian, G.; Haddow, J.E. The impact of maternal plasma DNA fetal fraction on next generation sequencing tests for common fetal aneuploidies. Prenat. Diagn. 2013, 33, 667-674. [CrossRef]

19. Ashoor, G.; Syngelaki, A.; Poon, L.C.Y.; Rezende, J.C.; Nicolaides, K.H. Fetal fraction in maternal plasma cell-free DNA at 11-13 weeks' gestation: Relation to maternal and fetal characteristics. Ultrasound Obstet. Gynecol. 2013, 41, 26-32. [CrossRef]

20. Wang, H.; Dong, Z.; Zhang, R.; Chau, M.H.K.; Yang, Z.; Tsang, K.Y.C.; Wong, H.K.; Gui, B.; Meng, Z.; Xiao, K.; et al. Low-pass genome sequencing versus chromosomal microarray analysis: Implementation in prenatal diagnosis. Genet. Med. 2020, 22, 500-510. [CrossRef]

21. Van Opstal, D.; Srebniak, M.I.; Polak, J.; de Vries, F.; Govaerts, L.C.; Joosten, M.; Go, A.T.; Knapen, M.F.; van den Berg, C.; Diderich, K.E.; et al. False Negative NIPT Results: Risk Figures for Chromosomes 13, 18 and 21 Based on Chorionic Villi Results in 5967 Cases and Literature Review. PLoS ONE 2016, 11, e0146794. [CrossRef] 
22. De Koning, M.A.; Haak, M.C.; Van Scheltema, P.N.A.; Peeters-Scholte, C.M.P.C.D.; Koopmann, T.T.; Nibbeling, E.A.R.; Aten, E.; den Hollander, N.S.; Ruivenkamp, C.A.L.; Hoffer, M.J.V.; et al. From diagnostic yield to clinical impact: A pilot study on the implementation of prenatal exome sequencing in routine care. Genet. Med. 2019, 21, 2303-2310. [CrossRef] [PubMed]

23. Jia, Y.; Zhao, H.; Shi, N.; Peng, W.; Xie, L.; Wang, W.; Jiang, F.; Zhang, H.; Wang, X. Genetic effects of a $13 q 31.1$ microdeletion detected by noninvasive prenatal testing (NIPT). Int. J. Clin. Exp. Pathol. 2014, 7, 7003-7011. [PubMed]

24. Jensen, T.J.; Dzakula, Z.; Deciu, C.; Boom, D.V.D.; Ehrich, M. Detection of Microdeletion 22q11.2 in a Fetus by Next-Generation Sequencing of Maternal Plasma. Clin. Chem. 2012, 58, 1148-1151. [CrossRef]

25. Mardy, A.; Wapner, R.J. Confined Placental Mosaicism and Its Impact on Confirmation of Nipt Results. Am. J. Med. Genet. Part C Semin. Med. Genet. 2016, 172, 118-122. [CrossRef]

26. Zhou, X.; Sui, L.; Xu, Y.; Song, Y.; Qi, Q.; Zhang, J.; Zhu, H.; Sun, H.; Tian, F.; Xu, M.; et al. Contribution of maternal copy number variations to false-positive fetal trisomies detected by noninvasive prenatal testing. Prenat. Diagn. 2017, 37, 318-322. [CrossRef]

27. Tamminga, S.; van Maarle, M.; Henneman, L.; Oudejans, C.B.; Cornel, M.C.; Sistermans, E.A. Maternal Plasma DNA and RNA Sequencing for Prenatal Testing. Adv. Clin. Chem. 2016, 74, 63-102. [CrossRef]

28. Xiang, Y.; Zhang, J.; Li, Q.; Zhou, X.; Wang, T.; Xu, M.; Xia, S.; Xing, Q.; Wang, L.; He, L.; et al. DNA methylome profiling of maternal peripheral blood and placentas reveal potential fetal DNA markers for non-invasive prenatal testing. Mol. Hum. Reprod. 2014, 20, 875-884. [CrossRef]

29. Martin, K.; Iyengar, S.; Kalyan, A.; Lan, C.; Simon, A.; Stosic, M.; Kobara, K.; Ravi, H.; Truong, T.; Ryan, A.; et al. Clinical experience with a single-nucleotide polymorphism-based non-invasive prenatal test for five clinically significant microdeletions. Clin. Genet. 2018, 93, 293-300. [CrossRef] [PubMed]

30. Vermeulen, C.; Geeven, G.; de Wit, E.; Verstegen, M.J.; Jansen, R.P.; van Kranenburg, M.; de Bruijn, E.; Pulit, S.; Kruisselbrink, E.; Shahsavari, Z.; et al. Sensitive Monogenic Noninvasive Prenatal Diagnosis by Targeted Haplotyping. Am. J. Hum. Genet. 2017, 101, 326-339. [CrossRef]

31. Chiu, R.W.K.; Chan, K.C.A.; Gao, Y.; Lau, V.Y.M.; Zheng, W.; Leung, T.Y.; Foo, C.H.F.; Xie, B.; Tsui, N.B.Y.; Lun, F.M.F.; et al. Noninvasive prenatal diagnosis of fetal chromosomal aneuploidy by massively parallel genomic sequencing of DNA in maternal plasma. Proc. Natl. Acad. Sci. USA 2008, 105, 20458-20463. [CrossRef]

32. Zhang, H.; Gao, Y.; Jiang, F.; Fu, M.; Yuan, Y.; Guo, Y.; Zhu, Z.; Lin, M.; Liu, Q.; Tian, Z.; et al. Non-Invasive Prenatal Testing for Trisomies 21, 18 and 13: Clinical Experience from 146958 Pregnancies. Ultrasound Obstet. Gynecol. 2015, 45, 530-538. [CrossRef]

33. Zhao, M.; Wang, Q.; Wang, Q.; Jia, P.; Zhao, Z. Computational tools for copy number variation (CNV) detection using nextgeneration sequencing data: Features and perspectives. BMC Bioinform. 2013, 14, 1-16. [CrossRef] [PubMed]

34. Liu, H.; Gao, Y.; Hu, Z.; Lin, L.; Yin, X.; Wang, J.; Chen, D.; Chen, F.; Jiang, H.; Ren, J.; et al. Performance Evaluation of NIPT in Detection of Chromosomal Copy Number Variants Using Low-Coverage Whole-Genome Sequencing of Plasma DNA. PLoS ONE 2016, 11, e0159233. [CrossRef] [PubMed]

35. Straver, R.; Sistermans, E.A.; Holstege, H.; Visser, A.; Oudejans, C.B.M.; Reinders, M.J.T. WISECONDOR: Detection of fetal aberrations from shallow sequencing maternal plasma based on a within-sample comparison scheme. Nucleic Acids Res. 2014, 42, e31. [CrossRef] [PubMed]

36. Raman, L.; Dheedene, A.; De Smet, M.; Van Dorpe, J.; Menten, B. WisecondorX: Improved copy number detection for routine shallow whole-genome sequencing. Nucleic Acids Res. 2019, 47, 1605-1614. [CrossRef]

37. Jiang, P.; Peng, X.; Su, X.; Sun, K.; Stephanie, C.Y.; Chu, W.I.; Leung, T.Y.; Sun, H.; Chiu, R.W.; Lo, Y.M.D.; et al. Fetalquant Sd: Accurate Quantification of Fetal DNA Fraction by Shallow-Depth Sequencing of Maternal Plasma DNA. NPJ Genom. Med. 2016, 1 , 1-7. [CrossRef]

38. Kim, S.K.; Hannum, G.; Geis, J.; Tynan, J.; Hogg, G.; Zhao, C.; Jensen, T.J.; Mazloom, A.R.; Oeth, P.; Ehrich, M.; et al. Determination of fetal DNA fraction from the plasma of pregnant women using sequence read counts. Prenat. Diagn. 2015, 35, 810-815. [CrossRef]

39. Peng, X.L.; Jiang, P. Bioinformatics Approaches for Fetal DNA Fraction Estimation in Noninvasive Prenatal Testing. Int. J. Mol. Sci. 2017, 18, 453. [CrossRef]

40. Straver, R.; Oudejans, C.B.M.; Sistermans, E.A.; Reinders, M.J.T. Calculating the fetal fraction for noninvasive prenatal testing based on genome-wide nucleosome profiles. Prenat. Diagn. 2016, 36, 614-621. [CrossRef]

41. Van der Meij, K.R.; Sistermans, E.A.; Macville, M.V.; Stevens, S.J.; Bax, C.J.; Bekker, M.N.; Bilardo, C.M.; Boon, E.M.; Boter, M.; Diderich, K.E.; et al. TRIDENT-2: National Implementation of Genome-wide Non-invasive Prenatal Testing as a First-Tier Screening Test in the Netherlands. Am. J. Hum. Genet. 2019, 105, 1091-1101. [CrossRef]

42. Li, H. Aligning Sequence Reads, Clone Sequences and Assembly Contigs with Bwa-Mem. arXiv 2013, arXiv:1303.3997.

43. Olshen, A.B.; Venkatraman, E.S.; Lucito, R.; Wigler, M. Circular binary segmentation for the analysis of array-based DNA copy number data. Biostatistics 2004, 5, 557-572. [CrossRef] [PubMed]

44. Talevich, E.; Shain, A.H.; Botton, T.; Bastian, B.C. CNVkit: Genome-Wide Copy Number Detection and Visualization from Targeted DNA Sequencing. PLoS Comput. Biol. 2016, 12, e1004873. [CrossRef] [PubMed]

45. Qin, Z.; Ljubimov, V.A.; Zhou, C.; Tong, Y.; Liang, J. Cell-free circulating tumor DNA in cancer. Chin. J. Cancer 2016, 35, 1-9. [CrossRef] [PubMed] 